\title{
COVERING SPACES OF PARACOMPACT SPACES
}

\author{
DAVID T. BROWN
}

ABSTRACT. This note proves that in the context of paracompact spaces, a definition used by the author implies the definition used by Saul Lubkin which does not involve the usual connectedness properties associated with covering spaces.

In preparing [1], the author modified a definition of a covering space given by S. Lubkin in [3] in order to work in the context of compact Hausdorff spaces. The purpose of this note is to show that in the more general context of paracompact spaces, the modified definition (Definition 1, below) implies Lubkin's definition.

Definition 1. Assume $X$ and $Y$ are paracompact spaces and $p$ is a continuous mapping of $X$ onto $Y$. We will say that $(X, p)$ is a covering space of $Y$ if:

(1) $\operatorname{card}\left(p^{-1}(y)\right)$ (= cardinality of $\left.p^{-1}(y)\right)$ is finite for each $y$ in $Y$;

(2) for each $y$ in $Y$ with $p^{-1}(y)=\left\{x_{1}, x_{2}, \ldots, x_{k}\right\}$, there exists an open neighborhood $V$ in $Y$ of $y$, and there exist disjoint neighborhoods $U_{1}, U_{2}, \ldots, U_{k}$ in $X$ of $x_{1}, x_{2}, \ldots, x_{k}$, respectively, such that $p^{-1}(V)=$ $\bigcup_{j=1}^{k} U_{j}$ and $p \mid U_{j}$ is a homeomorphism onto $V$ for $j=1,2, \cdots, k$.

Before presenting Lubkin's definition of a covering space, vre list his basic definitions. An entourage for a set $S$ is a subset $V$ of $S \times S$ which contains the diagonal. A contiguity for $S$, denoted by contig $(S)$, is a filter of entourages on $S \times S$. A base for $S$ is a filter base for contig( $S)$. If $S$ and $T$ are two sets each with contiguity, and if $f$ is a function from $S$ into $T$, then $f$ is called contiguous if and only if, for $V$ in contig(T), there exists $U$ in contig $(S)$ such that $f U \subset V f$ (i.e. $f(U[s]) \subset V[f(s)]$ for all $s$ in $S) .(U[s]=\{y \in S:(y, s) \in U\}$. $)$

If $\left\{A_{j}: j \in J\right\}$ is a family of subsets of $S$, and if $\left\{g_{j}: j \in J\right\}$ is a collection of functions such that $g_{j}$ maps $A_{j}$ into $T$, for each $j$, then $\left\{g_{j}: j \in J\right\}$ is called equiopen if $U \in$ contig $(S)$ implies there exists $V \in \operatorname{contig}(T)$

Received by the editors September 28, 1972 and, in revised form, October 12, 1973.

AMS (MOS) subject classifications (1970). Primary 54D99.

Key words and phrases. Covering space, paracompact space.

Copyright $\odot 1975$, American Mathematical Society 
such that for all $s \in A_{j}$ and $j \in J$,

$$
V\left[g_{j}(s)\right] \cap g_{j}\left(A_{j}\right) \subset g_{j}\left(U[s] \cap A_{j}\right) .
$$

Definition 2 (Lubkin [3]). Assume $S$ and $T$ are sets with contiguity and $p$ is a contiguous function from $S$ onto $T$. We will say that $(S, p)$ is an L-covering space of $T$ if $S$ has a base $\mathcal{U}$ of entourages such that for each $U \in \mathcal{U}$ there exists $V \in \operatorname{contig}(T)$ such that:

(a) $p \mid U[s]$ is an isomorphism of spaces from $U[s]$ onto $V[p(s)]$ for each $s \in S$. joint union.

(b) $p^{-1}(V[t])=\bigcup_{p(s)=t} U[s]$ for each $t \in T$, where the union is a dis-

(c) For each $t \in T$, the family of functions $\left\{p \mid U[s]: s \in p^{-1}(t)\right\}$ is equiopen.

Theorem. Assume $X$ and $Y$ are paracompact spaces and $p$ is a continuous mapping of $X$ onto $Y$. If $(X, p)$ is a covering space of $Y$, then $(X, p)$ is an L-covering space of $Y$.

Before proving our Theorem, we give a necessary and sufficient condition for $(X, p)$ to be a covering space of $Y$.

Proposition. Assume $X$ and $Y$ are paracompact spaces and $p$ is a continuous mapping of $X$ onto $Y$. Then $(X, p)$ is a covering space of $Y$ if and only if $p$ is an open mapping with the property that for each $y \in Y$, card $\left(p^{-1}(y)\right)$ is finite and for each $x \in X$, there exists a neighborhood $V_{x}$ of $x$ in $X$ such that $p \mid V_{x}$ is one-to-one.

Proof. Sufficiency is proved as in the corresponding case in $[1$, Theorem 1.1]. The usual arguments used for covering spaces show the necessity.

To prove the Theorem, assume $X$ and $p$ are given with $(X, p)$ a covering space of $Y$. Let contig $(X)$ denote the set of all nonempty neighborhoods -in $X \times X$ of the diagonal. (Equivalently, $V, \in$ contig $(X)$ if and only if for each $x \in X$, the set $V[x]$ is a neighborhood in $X$ of $x_{0}$ ) The filter $\operatorname{contig}(Y)$ is similarly defined. It follows that a function $f$ from $X$ into $Y$ is contiguous if and only if it is continuous.

Assume, for each $x \in X$, the set $T(x)$ is a subset of $X$ containing $x$. Our proof requires the following definitions concerning coverings of the form $\{T(x): x \in X\}$, which we will in the future abbreviate to $\{T(x)\}$.

Definition. We will say that the covering $\{T(x)\}$ satisfies I if:

(i) for any $y \in Y$, and $x$ and $x^{\prime}$ in $p^{-1}(y)$, we have $p(T(x))=p\left(T\left(x^{\prime}\right)\right)$.

(ii) $p^{-1}(p(T(x)))=\bigcup_{z \in p}-1(y) T(z)$. 
We will also say that the covering $\{T(x)\}$ satisfies II if, in addition to (i) and (ii) above, we have (iii) for each $x \in X$, the mapping $p \mid T(x)$ is a homeomorphism.

Note that (iii) implies that the union in (ii) is a disjoint union. Also, the hyputhesis that $(X, p)$ is a covering space of $Y$ ensures the existence of open coverings of $X$ which satisfy II.

Let $\mathcal{U}$ denote the collection of open sets $U \in \operatorname{contig}(X)$ such that the open covering $\{U[x]\}$ satisfies II.

Lemma 1. Assume $\{W(x)\}$ is an open covering of $X$ which satisfies II. Then there is an element $Z$ of $U$ such that the open covering $\{Z[x]\}$ is a refinement of $\{W(x)\}$.

Proof. Since a paracompact space is fully normal $[2$, p. 170], the open covering $\{W(x)\}$ has an open star refinement $\mathcal{C}$. Let $Z=\bigcup_{v \in \mathcal{O}} V \times V$. It is easily verified that $Z[x]=\bigcup_{x \in V} V$. By replacing $Z[x]$ by $Z[x] \cap$ $p^{-1}\left(\bigcap_{p\left(x^{\prime}\right)=p(x)} p\left(Z\left[x^{\prime}\right]\right)\right)$ if need be, we may, and do, assume the covering $\{Z[x]\}$ satisfies $\mathrm{I}$. (Recall that $p$ is an open mapping.) By the definition of a star refinement, for each $x \in X$, there is an $x^{\prime} \in X$ such that $Z[x] \subset W\left(x^{\prime}\right)$. It follows that the open covering $\{Z[x]\}$ satisfies II. This means $Z \in \mathcal{U}$ and the proof is complete.

Corollary. $\mathcal{U}$ is a base for contig $(X)$.

Given $U \in \mathcal{U}$, and $y \in Y$, define, for any $x \in p^{-1}(y), V(y)=p(U[x])$. $V(y)$ is a well-defined subset of $Y$ containing $y$. To complete the proof of our Theorem, we need only show condition (c) of Definition 2 is satisfied.

Lemma 2. If $U \in \mathcal{U}$, and $y_{0} \in Y$, then for any $W \in$ contig $(X)$, there exists $V \in \operatorname{contig}(Y)$ such that for any $x_{0} \in p^{-1}\left(y_{0}\right)$ and $z \in U\left[x_{0}\right]$,

$$
V[p(z)] \cap p\left(U\left[x_{0}\right]\right) \subset p\left(W[z] \cap U\left[x_{0}\right]\right) .
$$

Proof. We first assert the existence of a $T \in \mathcal{U}$ such that $\{T[x]\}$ is a refinement of $\{U[x]\}$, and $p \mid T[x] \cup T\left[x^{\prime}\right]$ is a homeomorphism whenever $x$ and $x^{\prime}$ are in $X$ such that $T[x] \cap T\left[x^{\prime}\right]$ is nonempty.

Let $\{R(x)\}$ be an open covering of $X$ which is a star refinement of $\{U[x]\}$ and which satisfies II. By Lemma 1 , there exists $T \in \mathcal{U}$ such that $\{T[x]\}$ is a refinement of $\{R(x)\}$. If $x, x^{\prime}$, and $x^{\prime \prime}$ are in $X$ such that $x \in$ $T\left[x^{\prime}\right] \cap T\left[x^{\prime \prime}\right]$, then for some $z^{\prime}$ and $z^{\prime \prime}$ in $X$,

$$
T\left[x^{\prime}\right] \cup T\left[x^{m}\right] \subset R\left(z^{\prime}\right) \cup R\left(z^{\prime \prime}\right) \subset \bigcup_{x \in R(t)} R(t)
$$


which is contained in $U\left[x_{0}\right]$ for some $x_{0} \in X$. Our assertion now follows from the fact that $U \in \mathcal{U}$.

To prove Lemma 2, we assume, without loss of generality, that $U \in \mathcal{U}$ has the property that $p \mid U[x] \cup U\left[x^{\prime}\right]$ is a homeomorphism whenever $U[x] \cap U\left[x^{\prime}\right]$ is nonempty. Let $Z \in \mathcal{U}$ be such that $Z \subset U \cap W$, and define $V \in$ contig $(X)$ by $V[y]=p(Z[x])$ for each $y \in Y$, where $x \in p^{-1}(y)$. The set $V[y]$ is a well-defined open neighborhood in $Y$ of $y$. For $x \in U\left[x_{0}\right]$ (*) $V[p(z)] \cap p\left(U\left[x_{0}\right]\right)=p(Z[z]) \cap p\left(U\left[x_{0}\right]\right) \subset p(U[z] \cap W[z]) \cap p\left(U\left[x_{0}\right]\right)$.

Since $U\left[x_{0}\right] \cap U[z] \neq \varnothing$, the mapping $p \mid U[z] \cup U\left[x_{0}\right]$ is a homeomorphism, and thus the right side of $(*)$ is a subset of

$$
\left.p\left(U[z] \cap W[z] \cap U\left[x_{0}\right]\right) \subset p(W[z]) \cap U\left[x_{0}\right]\right) .
$$

This completes the proof of Lemma 2 and also the proof of our Theorem.

\section{REFERENCES}

1. D. T. Brown, Galois theory for Banach algebras, Pacific J. Math. 30 (1969), 577-600. MR 40 \# 7809 .

2. J. L. Kelley, General topology, Van Nostrand, Princeton, N. J., 1955. MR 16, 1136.

3. S. Lubkin, Theory of covering spaces, Trans. Amer. Math. Soc. 104 (1962), 205-238. MR $25 \# 2581$.

DEPARTMENT OF MATHEMATICS, UNIVERSITY OF PITTSBURGH, PITTSBURGH, PENNSYLVANIA 15260

Current address: Department of Mathematics, Bethany College, Bethany, West Virginia 26032 